lopinavir/ritonavir and 53(66.2\%) patients received standard of care. Median age of patients in both groups were 8 years. Most patients have no co-morbidity. 16(59.3\%), 8(29.6\%) and $3(11.1 \%)$ patients in treatment group while 33(62.3\%), 20 $(37.7 \%)$ and none of patients in control group have upper respiratory infections, asymptomatic infection and pneumonia respectively. The median days from symptom onset to admission was 1 (IQR 0-2) in treatment and 3(IQR 1-5) in control group. The median days from symptom onset to start of lopinavir/ritonavir was $1(\mathrm{IQR} 1-3)$. The NPS viral load reduction from baseline to day 7 was greater in treatment group. Viral load dropped from mean $6.5 \log ^{10}$ copies/ml (SD 2.1) on admission to mean $4.6 \log ^{10}$ copies/ml (SD 2.1) on day 7 in patients treated with lopinavir/ritonavir. Viral load dropped from mean $6.4 \log ^{10}$ copies/ml (SD 1.8) on admission to mean $5.3 \log ^{10}$ copies/ml (SD 1.5) on day 7 in patient who did not receive lopinavir/ritonavir. However, the difference was not statistically significant. There was no significant difference in viral load on day 10 and day 14 between the two groups. There was also no significant difference in time to symptom resolution, PCR negativity or duration of hospitalization. No patient in the cohort require oxygen or intensive care. Among those received treatment, 7(25.9\%) patients experienced gastrointestinal symptoms possibly drug adverse effects with self-limited nausea, vomiting or diarrhea, as compared to $9(17 \%)$ patients in control group. 1(3.7\%) and $3(5.7 \%)$ patients in treatment and control group have mild deranged liver function. There was no significant difference in rate of gastrointestinal symptom or liver function derangement among the two groups. There was no QT interval prolongation or serious adverse effect noted in patients received treatment.

Conclusions There was no statistically significant difference in NPS viral load clearance, time to symptom resolution, PCR negativity and duration of hospitalization between paediatric COVID-19 patients treated with lopinavir/ritonavir and control. Self-limited gastrointestinal symptoms were common but use of lopinavir/ritonavir appeared to be safe in children with no serious side effect noted.

\section{PSYCHOLOGICAL ILL-EFFECTS AND RESILIENCE OF PROVIDERS OF CHILDREN WITH DEVELOPMENTAL DISABILITIES DURING THE COVID-19 PANDEMIC}

Ramkumar Aishworiya, Tammy SH Lim, Ying Qi Kang, Phyllis PL Lim, Shang Chee Chong, Mae Yue Tan. Singapore

\subsection{6/bmjpo-2021-RCPCH.195}

Background Providers of children with developmental disabilities (DD) had to rapidly innovate to translate face to face care to a virtual platform in order to continue to support the needs of their patients during the coronavirus 2019 pandemic. Objectives We aimed to explore the extent of psychological ill-effects, if any, and explore resilience in providers of children with DD.

Methods This was a voluntary cross-sectional study involving an anonymous questionnaire, administered via a secure online platform, between 13 May and 1 July 2020, coinciding with the Circuit Breaker (similar to a lockdown) period in Singapore. Participants included healthcare providers serving children with DD in various capacities at public and private healthcare settings in the country. Common diagnoses of DD included autism spectrum disorder and global developmental

\begin{tabular}{|c|c|c|c|c|c|c|}
\hline Variable & $\begin{array}{l}\text { Depression } \\
\text { (N) }\end{array}$ & $\%$ & $\begin{array}{l}\text { Anxiety } \\
\text { (N) }\end{array}$ & $\%$ & $\begin{array}{l}\text { Stress } \\
\text { (N) }\end{array}$ & $\%$ \\
\hline $\begin{array}{l}\text { Prevalence of positive screen } \\
\text { of traits on the DASS }\end{array}$ & 13 & 13.7 & 40 & 42.1 & 54 & 56.8 \\
\hline \multicolumn{7}{|c|}{ Among those who screened positive } \\
\hline Mild & 7 & 53.8 & 6 & 15 & 8 & 14.8 \\
\hline Moderate & 3 & 23.1 & 10 & 25 & 8 & 14.8 \\
\hline Severe & 1 & 7.7 & 6 & 15 & 9 & 16.7 \\
\hline Extremely Severe & 2 & 15.4 & 18 & 45 & 29 & 53.7 \\
\hline
\end{tabular}

delay. The Depression, Anxiety, and Stress Scales (DASS-21) and Connor-Davidson Resilience Scale 25 -item were administered. Descriptive statistics were conducted.

Results Ninety-five responses were received. Mean age of respondents was 37.4 years (SD 9.45), with $96.8 \%$ of them being female. A quarter (24.5\%) were medical professionals (doctor, nurse, psychologists), a third (33.0\%) were therapists (physiotherapists, speech therapists and occupational therapists), a third were school-based providers (teachers in mainstream or special-education schools, learning support educators), and the rest (8.5\%) were working in administrative aspects of dealing with children with DD (case manager, social workers, administrative staff). A quarter (25.5\%) worked in a hospital, 20.2\% worked in a school setting, $37.2 \%$ at a therapy centre (e.g early intervention centres), while $17.0 \%$ worked in social community agencies.

On the DASS (table 1), more than half $(56.8 \%$ ) of the respondents had a positive screen for stress, of which half of these (53.7\%) had levels which were in the 'extremely severe' range. Almost half (42.1\%) of providers had anxiety, of which $45.0 \%$ of these were 'extremely severe'. Depression rate was lower, with a positive screen present in $13.7 \%$ in this group of providers, and half of these were in the 'mild' range. Chi-square analysis showed that providers in schoolbased institutions were more likely than providers in nonschool-based institutions to have a positive screen for depression on the DASS-21 (36.8\% vs $8.0 \%, p=0.004)$. Mean resilience scores on the CD-RISC 25 was 62.7 (range 36.0 to 88.0 ), lower compared to the published normative data for this scale.

Conclusions Providers of children with DD face significant psychological ill-effects during this pandemic, as well as lower resilience compared to the general population. Targeted institutional support for these providers and interventions to improve their resilience are both crucial to enhance the mental well-being of these providers. This will in turn facilitate continued provision of care for children with DD despite the pandemic.

\section{OUTCOME OF FLASH GLUCOSE MONITORING- AN AUDIT}

Nikitha Rajaraman, Fathima Haniffa, Dhaara lyer. UK

\subsection{6/bmjpo-2021-RCPCH.196}

Background The use Flash Glucose Monitoring (FGM) is considered a useful technology for self-management of type 1 diabetes mellitus (T1DM). 
Patients will be required to meet specific criteria for initiation of FGM as per the National Institute of Clinical Excellence (NICE- NG 18) guidelines. Patients would also be required to meet specific measures of improvement in diabetic control for continuation of FGM under the National Health Service (NHS) funded services. These include completing regular scanning of sensor, sensor use time of more than $70 \%$, improvement in baseline $\mathrm{HbA} 1 \mathrm{c}$ and reduction in diabetes related hospital attendances or admissions.

Objectives To review the compliance and effectiveness of FGM use amongst paediatric patients with T1DM, within our service.

Methods This was a retrospective audit. A total of 46 patients were commenced on FGM between April 2019 and April 2020.

We collected and analysed the data, focusing on the percentage time of sensor use, number of scans per day, HbA1c at $0,1,3,6,9$ and 12 months and the average number of diabetes related hospital attendances or admissions per year before and after starting FGM.

Results Forty-six patients were started on FGM due to poor glucose monitoring, persistently high $\mathrm{HbA1c}$ and frequent hypoglycaemic episodes. The mean age of these children at the start of FGM was 12.8 years.

Of the total 46 patients, 28 (61\%) continued using FGM while the remaining 18 discontinued FGM. The reasons for discontinuing included primary skin conditions affecting adhesiveness of device (2 patients), changing to continuous glucose monitoring system (6 patients), nonengagement in the technology ( 2 patients) and personal choice (8 patients).

Out of these 28 patients, $18(64.3 \%)$ patients had sensor usage time of more than $70 \%$ and 8 (28.6\%) did more than 8 scans per day. On commencing FGM (0 months), the HbA1c of these 28 children ranged between 6.2 and $11.2 \mathrm{mmol} / \mathrm{mol}($ mean $=8.8)$. At 12 months, this was between 5.8 to $11.8 \mathrm{mmol} / \mathrm{mol}($ mean $=8.3)$, demonstrating a $0.5 \mathrm{mmol} / \mathrm{mol}$ reduction in the mean. A One-tailed Mann-Whitney U-test indicates that the HbA1c at 12 months is less than at 0 months, $U=279, p=0.07$. While this may not be statistically significant, we have demonstrated that after commencing FGM, 19 (67.9\%) patients' serial HbA1c displayed a downward trend, and 11 patients (39.2\%) had a reduction in diabetes related hospital attendances per year, with both criteria being met by 8 (28.5\%) patients.

Conclusions Our results demonstrate that FGM helps improve diabetes management and outcomes. The treating diabetes teams should continue to support patients in the use of advanced technology through regular follow-up, and empower patients and carers towards diabetes self-management, thereby improving outcomes.

\section{CASE SERIES OF CHILDREN PRESENTING WITH ACUTE VISUAL IMPAIRMENT}

Hnin Wint Wint Aung, Kyaw Linn. Myanmar

\subsection{6/bmjpo-2021-RCPCH.197}

Background Acute or rapidly progressive visual impairment in children is a medical emergency which needs early recognition and optimal management strategies. Among the different causes of acute visual impairment in children, optic neuritis $(\mathrm{ON})$ is an acquired disorder of the optic nerve due to inflammation, demyelination or degeneration and it is treatable. It may also represent a sentinel attack or relapse of a chronic demyelinating disorder such as multiple sclerosis (MS), neuromyelitis optica spectrum disorder (NMOSD) and myelin oligodendrocyte glycoprotein (MOG) antibody associated diseases.

Objectives This is to report a case series of three children presenting with acute visual impairment due to optic neuritis.

Methods This is a retrospective, descriptive case series by reviewing medical records of initial admission and follow-up.

Results Three patients were included in this case series.

Case 1: a 6-year-old girl suffered from acute visual impairment which was started on the right side and then involved bilaterally. Other systemic and neurological examinations were normal. Visual acuity was just perception of light on both eyes. She had a previous episode of acute disseminated encephalomyelitis (ADEM) 4 months ago. Investigations revealed positive MOG-IgG with negative anti-aquaporin 4 (AQP4) antibodies. MRI brain showed T2 hyperintense lesions with contrast enhancement of bilateral optic nerves. She was diagnosed as MOG-optic neuritis. Her vision fully recovered with intravenous methylprednisolone and immunoglobulin.

Case 2: a 10-year-old girl presented with a relapsing bilateral optic neuritis. At the first presentation, her visual acuity was perception to light only on both eyes. Anti-aquaporin 4 antibody was positive and bilateral optic nerves enlargement with contrast enhancement was seen in MRI brain. Her vision almost fully recovered with intravenous methylprednisolone. She had relapsed with acute visual impairment, intractable hiccups and vomiting, 3 months later. She was diagnosed as bilateral optic neuritis with area postrema syndrome; therefore, the second cycle of intravenous methylprednisolone was started again together with mycophenolate mofetil for longterm immunosuppression. However, her vision only partially recovered with visual acuity $6 / 20$ on the right side and $6 / 60$ on the left.

Case 3: a 7.5-year-old boy presented with acute onset of bilateral visual impairment with sensory impairment below T4 level. His blood inflammatory markers were normal and antiaquaporin 4 and anti-MOG antibodies were also negative. Features of bilateral optic neuritis and transverse myelitis in spinal cord from C4 to T5 were seen in MRI studies. The diagnosis was NMOSD with negative AQP4-IgG. His vision also fully recovered after receiving intravenous methylprednisolone therapy.

Conclusions Optic neuritis should always be considered in children with acute visual impairment. Complete neurological and ophthalmological examinations are essential features of the clinical evaluation. Ophthalmology referral is required. Magnetic resonance imaging (MRI) study of the brain and orbits with gadolinium contrast provides confirmation of the diagnosis and underlying causes in most cases. Other tests including lumbar puncture, antibody testing for MOG and AQP4 antibodies are also recommended. Accurate and early diagnosis of optic neuritis is crucial as prompt aggressive treatment may result in a good prognosis on vision and mitigation of long-term disability. 